\title{
Resource Review
}

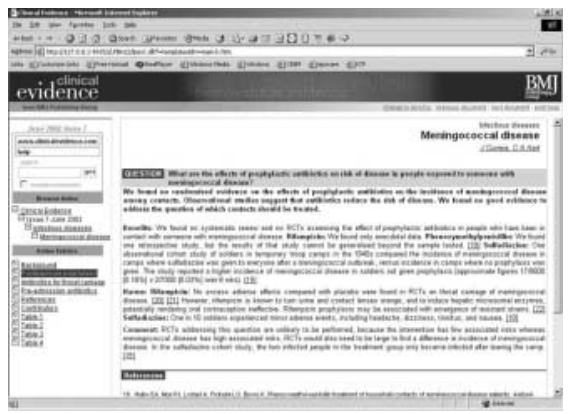

Clinical Evidence Concise. London: BMJ Publishing Group, 2002.
Now in its 7th issue, Clinical Evidence has undergone a rapid expansion in the past 3 years. Covering 158 clinical problems, this text serves as a resource for primary care clinicians to access the current best evidence supporting therapeutic interventions. To function as a "first point of call" for clinicians assessing treatment options, Clinical Evidence has devised a concise text version with an accompanying CD-ROM. Clinical Evidence Concise provides a summary of the most important information found in the full length version.

The evidence in Clinical Evidence Concise is compiled using comprehensive search, retrieval, and appraisal protocols. Topics are selected using national databases of morbidity, mortality, and consultation rates, in conjunction with the advice of primary care clinicians and patient groups. Using a rigorous search protocol, the Cochrane Library, Medline, and EMBASE/Excerpta Medica are searched for answers to the outlined questions. Good quality systematic reviews are initially sought, but if these are not found, primary randomised controlled trials are assessed. All articles are appraised using validated methodological criteria. The evidence is then assembled, summarised, and reviewed by panels of 3 expert clinicians with experience in clinical epidemiology. It is updated and expanded every 6 months, with complete topic revisions every 8 months. The evolution of Clinical Evidence Concise with respect to topics, questions, and format is guided by feedback from users.

This work encompasses a broad range of medical disorders, including cardiovascular; digestive; renal; endocrine; infectious; neurologic, ear, nose, and throat; eye; and respiratory diseases, in addition to mental, sexual, men's, women's, and children's health. Other topics include wounds, perioperative care, poisoning, and pregnancy and childbirth management. The full text comprehensively addresses each topic and the quality of the evidence, but the concise version highlights only key information. The therapies are categorized as effective or ineffective, and specific interventions as beneficial or harmful based on the availability and quality of the evidence. Answers include comments on the nature of the evidence (eg, systematic review $v$ randomised trial), the study population characteristics, and whether the intervention was compared with placebo. Treatment effectiveness is presented as the number needed to treat in Clinical Evidence Concise. Links to the complete citations are provided on the accompanying CD-ROM. The concise version also includes icons to indicate the presence of relevant tables, figures, and glossary terms found on the CD-ROM or in the full length text.

The brief summary of information that is provided makes Clinical Evidence Concise easy to use. The accompanying CD-ROM can be used to delve further into the answers when time and interest permit. It is also easily portable-something not possible with the full text version.

Although the number of topics covered in Clinical Evidence has nearly tripled since its inception, the scope is still limited and may not cover the patient or situation at hand. Furthermore, only therapy and prevention are addressed; evidence for diagnosis and prognosis is not covered.

With the brief information provided, one must also be conscious of the confines of directly applying this information in clinical care. This is especially true in interpreting numbers needed to treat. These must be modified to fit the individual patient's circumstances. Thus, although both the full length and concise versions are useful, it is up to individual clinicians to translate the evidence into estimates of effectiveness for their own patients.

GLORIA RAMBALDINI, MD University of Toronto Toronto, Ontario, Canada

Ratings:

Methods: $\star \star \star \star \star$

Clinical usefulness: $\star \star \star \star \star$ i 